\title{
Design, Synthesis and Structure-Activity Relationships of a Phenotypic Small Library against Protozoan Infections ${ }^{\dagger}$
}

\author{
Elisa Uliassi ${ }^{1}$, Lorna Piazzi ${ }^{1}$, Federica Belluti ${ }^{1}$, Marcel Kaiser ${ }^{2,3}$, Reto Brun ${ }^{2,3}$, Sheraz Gul ${ }^{4}$, \\ Bernhard Ellinger ${ }^{4}$, Carolina B. Moraes ${ }^{5}$, Lucio H. Freitas-Junior ${ }^{5}$, Chiara Borsari ${ }^{6}$, \\ Maria Paola Costi ${ }^{6}$ and Maria Laura Bolognesi ${ }^{1, *}$ \\ 1 Department of Pharmacy and Biotechnology, Alma Mater Studiorum-University of Bologna, \\ Via Belmeloro 6, 40126 Bologna, Italy; elisa.uliassi3@unibo.it (E.U.); lpiazzi@iciq.es (L.P.); \\ federica.belluti@unibo.it (F.B.) \\ 2 Swiss Tropical and Public Health Institute, 4002 Basel, Switzerland; marcel.kaiser@unibas.ch (M.K.); \\ reto.brun@unibas.ch (R.B.) \\ 3 University of Basel, Petersplatz 1, 4003 Basel, Switzerland \\ 4 Fraunhofer Institute for Molecular Biology and Applied Ecology Screening Port, D-22525 Hamburg, \\ Germany; Sheraz.Gul@ime.fraunhofer.de (S.G.); Bernhard.Ellinger@ime.fraunhofer.de (B.E.) \\ 5 Laboratório Nacional de Biociências (LNBio), Centro Nacional de Pesquisa em Energia e Materiais \\ (CNPEM), SP13083-100 Campinas, Brazil; carolinaborsoi@gmail.com (C.B.M.); \\ lucio.freitas@butantan.gov.br (L.H.F.-J.) \\ 6 Department of Life Sciences, University of Modena and Reggio Emilia, Via Campi 103, 41125 Modena, Italy; \\ chiara.borsari@unibas.ch (C.B.); mariapaola.costi@unimore.it (M.P.C.) \\ * Correspondence: marialaura.bolognesi@unibo.it \\ † Presented at the 1st Molecules Medicinal Chemistry Symposium, Barcelona, Spain, 8 September 2017. \\ Published: 19 October 2017
}

Protozoan infections (Plasmodium spp., Leishmania spp., and Trypanosoma spp.) remain one of the most pressing global health concerns, affecting billions of people and producing unsustainable economic burdens [1]. Current pharmacotherapy is inadequate, and appropriate technologies should be exploited to identify novel drug candidates in a cost- and time-effective manner. Accordingly, an effective strategy could exploit privileged structures to generate libraries of high-quality compounds, combined with the feasibility of a phenotypic assay, and the early evaluation of the ADME-tox profile.

On these bases, we generated an 18-membered combinatorial library by fast assembling phenothiazine, biphenyl and phenylpiperazine anti-protozoan privileged scaffolds via a Huisgen cycloaddition. Thanks to NMTrypI [2] and SPHTI [3] screening facilities, we tested 1-18 against T. brucei and cruzi, L. infantum and donovani, and P. falciparum, and counter-screened selectivity against mammalian cells (L6 and A549). In parallel, ADME-tox properties were assessed by testing hERG, CYP inhibition, and mitochondrial viability.

Despite the small number of synthesized compounds, this strategy led to the successful identification of interesting hits with promising profiles. Particularly, $\mathbf{4}$ and $\mathbf{9}$ showed IC 50 values of 3.8 and $3.4 \mu \mathrm{M}$ against $T$. cruzi, together with an excellent selectivity (SI (IC50 (L6)/IC $50(T c)$ ) $>48$ and $\%$ A549 cell growth at $10 \mu \mathrm{M}>100 \%)$.

Acknowledgments: We acknowledge the University of Bologna (RFO), MIUR (PRIN 201274BNKN_003), and NMTrypI European project (under Grant Agreement $n^{\circ}$ 603240) for the anti-trypanosomatid and early-tox profiling. No additional funds have been received to publish in open access.

Author Contributions: M.L.B. designed the research project. M.L.B. and E.U. analyzed data. E.U., L.P. and F.B. afforded synthesis and characterization of compounds. C.B. and M.P.C. annotated the compounds and 
conceived the experiments. M.K., R.B., C.B.M. and L.H.F.-J. performed whole-cell assays. S.G. and B.E. carried out the early tox-profiling.

Conflicts of Interest: The authors declare no conflict of interest.

\section{References}

1. Field, M.C.; Horn, D.; Fairlamb, A.H.; Ferguson, M.A.; Gray, D.W.; Read, K.D.; De Rycker, M.; Torrie, L.S.; Wyatt, P.G.; Wyllie, S.; Gilbert, I.H. Antitrypanosomatid Drug Discovery: An Ongoing Challenge and a Continuing Need. Nat. Rev. Microbiol. 2017, 15, 217-231.

2. The Anti-Parasitic Activity and the Early-Toxicity Profiling of the Compounds Were Developed within the International Collaborative Effort of the European Union's Seventh Framework Programme under Grant Agreement $\mathrm{n}^{\circ} 603240$ (NMTrypI-New Medicines for Trypanosomatidic Infections). Available online: http://fp7-nmtrypi.eu (accessed on 16 October 2017).

3. SPHTI-Swiss Tropical \& Public Health Institute, Parasite Chemotherapy Unit. Available online: https://www.swisstph.ch/en/ (accessed on 16 October 2017).

(C) 2017 by the authors. Licensee MDPI, Basel, Switzerland. This article is an open access article distributed under the terms and conditions of the Creative Commons Attribution (CC BY) license (http://creativecommons.org/licenses/by/4.0/). 